\title{
Targeted resequencing of phosphorus metabolism-related genes in 86 patients with hypophosphatemic rickets/osteomalacia
}

\author{
JIEMEI GU, CHUN WANG, HAO ZHANG, HUA YUE, WEIWEI HU, \\ JINWEI HE, WENZHEN FU and ZHENLIN ZHANG
}

\begin{abstract}
Metabolic Bone Disease and Genetic Research Unit, Department of Osteoporosis and Bone Diseases, Shanghai Key Clinical Center for Metabolic Disease, Shanghai Jiao Tong University Affiliated Sixth People's Hospital, Shanghai 200233, P.R. China
\end{abstract}

Received February 16, 2018; Accepted June 8, 2018

DOI: $10.3892 / \mathrm{ijmm} .2018 .3730$

\begin{abstract}
Hypophosphatemic rickets/osteomalacia is characterized by defective renal phosphate reabsorption and abnormal bone mineralization. Hypophosphatemic rickets/osteomalacia consists of inherited and acquired forms, many of which have unknown aetiology. In the present study, next-generation sequencing-based resequencing was used on samples from Chinese subjects with hypophosphatemic rickets/osteomalacia, aiming to detect the spectrum of pathogenic genes in these patients. A total of 86 hypophosphatemic rickets/osteomalacia patients (ranging from 3 to 70 years old) were recruited. Patients with tumour-induced osteomalacia (TIO), renal tubular acidosis, renal osteodystrophy, and adefovir-induced Fanconi syndrome were excluded. Targeted massively parallel resequencing of 196 candidate genes for hypophosphatemic rickets/osteomalacia was performed in the 86 affected unrelated individuals (cases) and in 100 unrelated healthy controls to identify new genes and mutations in known genes that cause hypophosphatemic rickets/osteomalacia. The results identified seven phosphate-regulating gene with homologies to endopeptidases on the $\mathrm{X}$ chromosome ( $P H E X)$ mutations (of which two were novel) and one novel dentin matrix protein 1 $(D M P 1)$ mutation in eight patients. Following targeted exome sequencing data analysis, 14 candidate disease-related gene loci were selected, two of which were of most concern regarding disease severity. Further validation of the present results is warranted, with additional sequencing projects and functional tests. To our knowledge, the present study is the largest cohort of cases with hypophosphatemic rickets/osteomalacia to undergo targeted resequencing. The diagnosis and understanding of the molecular aetiologies of these disorders will be improved by this fast and efficient approach.
\end{abstract}

Correspondence to: Dr Zhenlin Zhang, Metabolic Bone Disease and Genetic Research Unit, Department of Osteoporosis and Bone Diseases, Shanghai Key Clinical Center for Metabolic Disease, Shanghai Jiao Tong University Affiliated Sixth People's Hospital, 600 Yi-shan Road, Shanghai 200233, P.R. China

E-mail: zhangzl@sjtu.edu.cn

Key words: targeted sequencing, genes, rickets, hypophosphatemic, osteomalacia

\section{Introduction}

Hypophosphatemic rickets/osteomalacia consists of inherited and acquired forms that share a common pathophysiology and clinical features (1). In hereditary cases, X-linked hypophosphatemic rickets, due to inactivating mutations in the gene encoding phosphate regulating gene with homologies to endopeptidases on the X chromosome (PHEX; MIM 300550), is the most common (2). To date, $>339$ different PHEX mutations have been described in patients with hypophosphatemic rickets; these mutations include nonsense mutations, missense mutations, splicing site mutations, insertions, and deletions in different positions (http://www.phexdb.mcgill.ca/; accessed January 2018). Subsequently, mutations in several other genes responsible for rare forms of hypophosphatemic rickets/osteomalacia have been identified (Table I) (3-12).

A specific molecular diagnosis of hypophosphatemic rickets/osteomalacia can help clinical diagnosis, improve the mental health of patients, direct therapeutic interventions, including reproductive counselling, and provide a better understanding of disease pathogenesis $(13,14)$. However, it has been difficult to determine the specific mutations in hypophosphatemic rickets/osteomalacia because of their extensive genetic heterogeneity, which so far involves 7 known genes and potentially many more unknown genes. The genetic backgrounds overlap, and clinical ambiguities for some types of hypophosphatemic rickets/osteomalacia further increase the difficulties in determining causative mutations, because no specific panel of hypophosphatemic rickets/osteomalacia genes can be predicted in these circumstances.

Detection of specific mutations among many candidate genes by conventional techniques is challenging. Two standard methods, homozygosity mapping and linkage analysis, used to localize a genetic defect, require either a sufficient number of informative meiotic events in a family or a number of individual families with similar phenotypes. Sanger sequencing can be used for mutation screening, but if a large number of genes need to be tested, it is often expensive and time-prohibitive. Compared with these approaches, next-generation massively parallel sequencing (MPS) with various applications offers high sensitivity and rate of variation detection and is currently considered an efficient method for gene mutation screening (15-17). 
In the present study, 86 Chinese patients with hypophosphatemic rickets/osteomalacia were recruited, excluding patients with Fanconi syndrome, renal tubular acidosis, chronic renal failure or tumour-induced osteomalacia (TIO). Then, genetic studies were performed to investigate the pathogenic genes in these patients. A novel testing panel was designed and validated using a single diagnostic platform to facilitate the sequencing of multiple genes that may cause some of the common forms of hypophosphatemic rickets/osteomalacia. The panel included 196 target genes implicated in hypophosphatemic rickets/osteomalacia.

\section{Materials and methods}

Subjects. The present study was approved by the Ethics Committee of the Shanghai Jiao Tong University-Affiliated Sixth People's Hospital [Shanghai, China; nos. 2013-87 and 2014-KY-001(k)]. All the subjects were Han Chinese and recruited by the Department of Osteoporosis and Bone Diseases; signed informed consent was obtained from the subjects prior to participation in the study. Written informed consent and permissions were obtained from the parents on behalf of the minors/child participants involved in the study. Consent to publish was also obtained from all the participants regarding the reporting of these patients' data.

The subjects were enrolled in the study at the Department of Osteoporosis and Bone Diseases in Shanghai Jiao Tong University Affiliated Sixth People's Hospital between October 2014 and July 2017. Eighty-six patients with hypophosphatemic rickets/osteomalacia and 100 unrelated, healthy, ethnic-matched controls (50 males and 50 females, $64.5 \pm 9.1$ years old, Chinese Han ethnicity) were examined in the present study. Among the 86 patients with hypophosphatemic rickets/osteomalacia, seven had a family history of the disease (Fig. 1). Twelve of the hypophosphatemic rickets patients were $<18$ years old, with an average age of $10.4 \pm 5.6$ years. Seventy-four of the osteomalacia patients were $>18$ years of age, with an average age of $42.1 \pm 15.5$ years. The clinical manifestations of these hypophosphatemic rickets/osteomalacia patients included short stature, bone pain, lower extremity deformities, calcification of the ligaments, and dental abnormalities. The serum phosphate levels of the majority of patients were $<0.7 \mathrm{mmol} / 1$ (normal range is $0.8-1.6 \mathrm{mmol} / 1$ for adults and $1.29-1.94 \mathrm{mmol} / 1$ for patients aged 2-18 years), and serum alkaline phosphatase levels exhibited by the majority of patients were $>160 \mathrm{u} / \mathrm{l}$ (normal range is $15-112 \mathrm{u} / \mathrm{l}$ for adults and $58-400 \mathrm{u} / \mathrm{l}$ for patients aged from 2-18 years) (18). The principal characteristics of these patients are listed in Table II.

A total of 186 DNA samples were extracted from peripheral white blood cells obtained from each of the subjects using conventional methods. Medical histories were obtained, and physical, audiological, and radiological examinations were performed for the subjects. Serum fibroblast growth factor 23 (FGF23) levels were measured for all the patients. ${ }^{18} \mathrm{~F}$-fluorodeoxyglucose positron emission tomography and computed tomography $\left({ }^{18} \mathrm{~F}-\mathrm{FDG}\right.$ PET/CT $)$ and $99 \mathrm{mTc}$-octreotide $(99 \mathrm{mTc}-\mathrm{OCT})$ scintigraphy were performed on 10 and 20 of the patients, respectively. These patients were diagnosed with hypophosphatemic rickets/osteomalacia based on their clinical
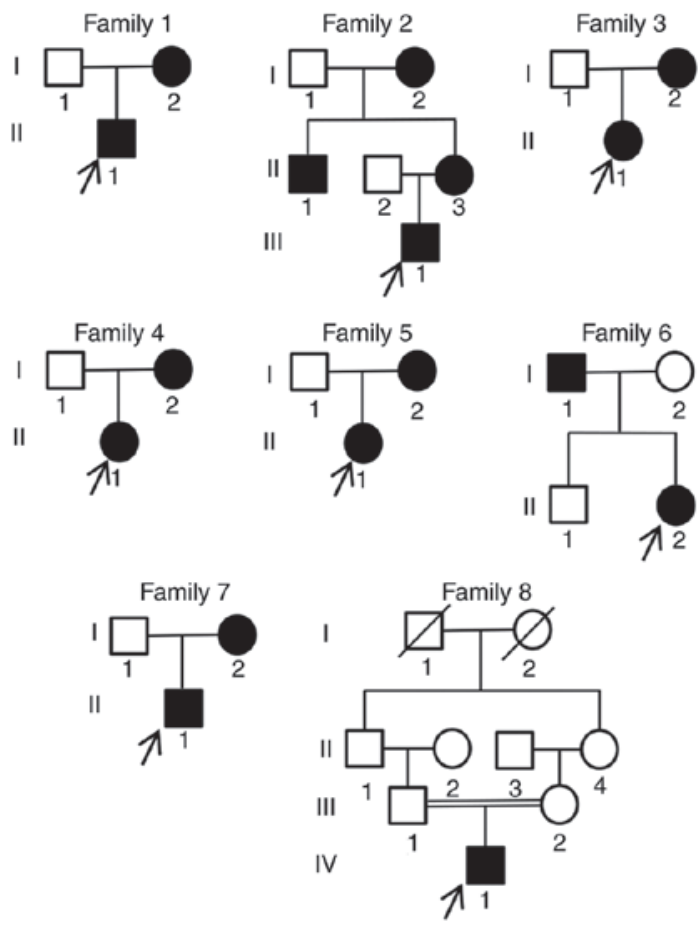

Figure 1. Pedigree for familial patients with hypophosphatemic rickets/osteomalacia. Black symbols represent the affected individuals, and open symbols represent the unaffected individuals. Circles and squares represent females and males, respectively. The arrows identify the probands in the families.

features, medical history, physical examination, plain radiography, radionuclide bone scan, elevated alkaline phosphatase (ALP) levels, and normal ${ }^{18} \mathrm{~F}$-FDG PET/CT and $99 \mathrm{mTc}-\mathrm{OCT}$ results. Acquired hypophosphatemic rickets/osteomalacia patients were not included in the present study, such as those with TIO, renal tubular acidosis, renal osteodystrophy, and adefovir-induced Fanconi syndrome.

Candidate gene selection. A total of 196 genes were selected for the target gene capture presented in the current study. These genes were selected after exploring public Internet databases, such as PubMed. Genes that may affect phosphate homeostasis and skeletal mineralization were selected. Key words, including 'PTH related genes', 'FGF23 related genes', 'Phosphate transporters', 'Osteoblast related genes', 'Wnt signalling pathway related genes' and 'vitamin D pathway genes', were included in the search. The Gene Expression Omnibus (GEO) profiles tool from the National Center for Biotechnology Information (NCBI) was used to evaluate expression patterns of the selected genes. All of the genes designated in the targeted gene capture sequencing are listed in Table III.

DNA extraction and next-generation sequencing. Samples of $2 \mathrm{ml}$ peripheral blood were obtained from the individuals. QIAamp DNA blood kits (Qiagen GmbH, Hilden, Germany) were used to isolate genomic DNA from the blood samples, according to the manufacturer's standard procedure. Then, DNA purity was tested by calculating the ratio of the absorbance measurements at 260 and $280 \mathrm{~nm}$, using an Invitrogen Qbit spectrophotometer. The genomic DNA was sheared using 
Table I. Hypophosphatemic rickets/osteomalacia caused by gene mutations.

\begin{tabular}{lcccc}
\hline Classification & Abbreviation & MIM no. & Location & Gene \\
\hline X-linked hypophosphatemic rickets & XLHR & 307800 & Xp22.1 & $P H E X[2]$ \\
Autosomal dominant hypophosphatemic rickets & ADHR & 193100 & $12 \mathrm{p} 13.32$ & $F G F 23[3]$ \\
Autosomal recessive hypophosphatemic rickets 1 & ARHR1 & 241520 & $4 \mathrm{q} .22 .1$ & $D M P 1[4,5]$ \\
Autosomal recessive hypophosphatemic rickets 2 & ARHR2 & 613312 & $6 \mathrm{q} 23.2$ & $E N P P 1[6,7]$ \\
Raine syndrome & & 259775 & $7 \mathrm{p} 22.3$ & $F A M 20 c[8]$ \\
Hereditary hypophosphatemic rickets with hypercalciuria & HHRH & 241530 & $9 \mathrm{q} 34.3$ & SLC34A3 [9,10] \\
X-linked recessive hypophosphatemic rickets & & 300554 & Xp11.23-p11.22 & CLCN5 [11,12] \\
\hline
\end{tabular}

PHEX, phosphate-regulating gene with homologies to endopeptidases on the X chromosome; FGF23, fibroblast growth factor 23; DMP1, dentin matrix protein 1; ENNP1, ectonucleotide pyrophosphatase/phosphodiesterase 1; FAM20c, family with sequence similarity 20 member C; SLC34A3, solute carrier family 34 member C; CLCN5, chloride voltage-gated channel 5.

Table II. Clinical features of the patients.

\begin{tabular}{lc}
\hline Clinical feature & Patients \\
\hline Age, years (median) $^{\mathrm{a}}$ & $34(21-50)$ \\
Age at onset, years (median) $^{\mathrm{a}}$ & $25(1-31.5)$ \\
Number of patients $\leq 18$ years old & 12 \\
Sex & Male: $42 /$ female: 44 \\
Height, cm & $151.96 \pm 8.7$ \\
Body weight, kg & $54.17 \pm 11.41$ \\
Serum Ca, mmol/1 & $2.27 \pm 0.1$ \\
Serum P, mmol/1 & $0.49 \pm 0.15$ \\
ALP, u/l (median)a & $265(177-378)$ \\
FGF23 level, RU/ml (median) ${ }^{\mathrm{a}}$ & $38.29(22.09-89.55)$ \\
$99 \mathrm{mTc}-\mathrm{TOC}$ & Normal $(20$ patients were \\
& examined $)$ \\
${ }^{18}$ F-FDG PET/CT & Normal $(10$ patients were \\
Family histories & examined $)$ \\
\hline
\end{tabular}

The normal ranges for serum ALP, Ca, P and PTH in adults are $15-112 \mathrm{U} / \mathrm{l}, 2.08-2.60$ and $0.80-1.60 \mathrm{mmol} / \mathrm{l}$, respectively. The normal ranges for serum ALP and $\mathrm{P}$ in patients aged 2-18 years old are 58-400 U/l and 1.29-1.94 mmol/l, respectively. ${ }^{a}$ Quartiles (Q1 and Q3) were used to describe these data. ALP, alkaline phosphatase; FGF23, fibroblast growth factor 23; 99mTc-TOC, 99mTc-octreotide; ${ }^{18} \mathrm{~F}$-FDG PET/CT, ${ }^{18} \mathrm{~F}$-fluorodeoxyglucose positron emission tomography/computed tomography.

a Covaris system. Then, the sample preparation was completed using the Truseq DNA Sample Preparation kit (Illumina, Inc., San Diego, CA, USA).

Custom capture oligonucleotides were designed using the SureDesign website from Agilent Technologies, Inc. (Santa Clara, CA, USA). The hybridization reactions were performed on an AB2720 Thermal Cycler (Thermo Fisher Scientific, Inc., Waltham, MA, USA) with the following hybridization conditions: The hybridization mixture was incubated for 16 or $24 \mathrm{~h}$ at $65^{\circ} \mathrm{C}$ with a heated lid at $105^{\circ} \mathrm{C}$, and following hybridization the hybridization mixture was captured and washed with magnetic beads (Invitrogen; Thermo Fisher Scientific, Inc.) and the SureSelect Target Enrichment kit (Agilent Technologies, Inc.).

The capture products were enriched under the following cycling conditions: $98^{\circ} \mathrm{C}$ for $30 \mathrm{sec}$, followed by 10 cycles of $98^{\circ} \mathrm{C}$ for $10 \mathrm{sec}, 60^{\circ} \mathrm{C}$ for $30 \mathrm{sec}$, and $72^{\circ} \mathrm{C}$ for $30 \mathrm{sec}$, and a final step of $72^{\circ} \mathrm{C}$ for $5 \mathrm{~min}$. Several of the libraries were pooled, and then, bridge amplification was performed using a cBot (Illumina, Inc.), according to the manufacturer's standard cluster generation protocols. Following hybridization of the sequencing primer, base incorporation was conducted using a HiSeq Sequencer (Illumina, Inc.), according to the manufacturer's standard sequencing protocols, for 101 cycles of sequencing per read to generate the paired-end reads, which included $100 \mathrm{bps}$ at each end and $6 \mathrm{bps}$ of the index tag.

One hundred and ninety-six gene coding and UTR regions, 2,000 promoter regions and $25 \mathrm{bp}$ from the splice site probes were designed. The total probe length was $1 \mathrm{M}$, with the number 15099.

Targeted sequencing data analysis. Targeted sequencing was performed by the Center for Genetic \& Genomic Analysis, Genesky Biotechnologies Inc. (Shanghai, China). All 186 samples were sequenced together to an average coverage of 101.5x for each sample (median 263.575 Mb per sample). The mean target rate with at least $20 \mathrm{X}$ is $88.30 \%$, the mean target rate with at least $10 \mathrm{X}$ is $93.06 \%$, and the mean target rate with at least $1 \mathrm{X}$ is $98.34 \%$. Paired-end sequencing was conducted for 100 bases from each end of the 200 -bp insert fragment libraries using standard Illumina protocols, and each read was aligned to hg19 (February 2009 GRCh37/hg19) from the University of California Santa Cruz (UCSC) Genome Browser using Burrows-Wheeler Aligner (BWA) with default parameters. Polymerase chain reaction (PCR) duplicates with the same start site for both ends were removed using Picard tools. Variants were called using Genome Analysis Toolkit (GATK) 1.6 tools. The variant quality score recalibration (VQSR) method, which builds an adaptive error model using known variant sites, was applied to estimate the probability that each variant is a true genetic variant or a machine artefact. The variants were annotated using functional annotation of genetic variants from high-throughput sequencing data, and all of the 
Table III. Genes designated in the targeted gene capture sequencing.

Genes

PDPN, ALPL, C1orf31, TARBP1, CDC42, CYP2J2, ZZZ3, USP33, PSRC1, CTSK, LCE5A, RSU1, CUBN, LOC100506939, DKK1, CYP2C9, PPRC1, NOLC1, FGFR2, ACADSB, PTH, CYP2R1, CD44, SNX32, CFL1, LRP5, PPP6R3, FGF4, FGF3, FLJ42102, DHCR7, NADSYN1, KRTAP5-7, WTAPP1, MMP1, HYOU1, FGF23, MIR1244-1, LRP6, MGP, LOC400027, ARID2, SCAF11, WNT10B, WNT1, KRT4, KRT79, GPR84, METTL7B, ITGA7, BLOC1S1-RDH5, RDH16, CYP27B1, IGF1, COL4A1, COL4A2, COL4A2-AS1, EIF2AK4, SRP14, IGDCC3, IGDCC4, CCDC33, LOC729739, CYP11A1, CYP1A1, GDPD3, MAPK3, MIR4733, NF1, OMG, EVI2A, RAB11FIP4, SOST, AXIN2, SLC9A3R1, MIR3615, NAT9, OTOP3, ITGB4, GALK1, MBD1, CXXC1, STARD6, C18orf54, CCL25, FBN3, MBD3L1, MUC16, OLFM2, COL5A3, RDH8, KANK2, C19orf80, DOCK6, CD97, DDX39A, CPAMD8, HAUS8, MYO9B, BABAM1, ANKLE1, ZNF814, MIR4426, MTIF2, CAPG, FLJ42351, SLC20A1, GALNT3, PTH2R, CYP27A1, PRKAG3, COL6A3, CYP24A1, GNAS, CXADR, BTG3, YPEL1, MAPK1, PDGFB, PTH1R, IGSF11-AS1, IGSF11, C3orf30, IGSF10, OTOL1, FGFR3, OTOP1, NCAPG, PDGFRA, GC, DSPP, DMP1, IBSP, MEPE, SPP1, DKK2, USP53, C4orf3, FGF2, NUDT6, SCOC, CLGN, FGF1, CSF1R, PDGFRB, SLC34A1, PFN3, SLC17A4, SLC17A1, SLC17A2, ZNF184, RUNX2, SUPT3H, KIAA1919, REV3L, TRAF3IP2-AS1, ENPP1, CTGF, FAM20C, LOC100288524, PDGFA, FLJ44511, SFRP4, ERDR1, DMTF1, C7orf23, CYP3A7-CYP3AP1, CYP3A4, PRSS3P2, PRSS2, TRPV6, TRPV5, CTSB, PDGFRL, MTUS1, BMP1, PHYHIP, LETM2, FGFR1, SFRP1, VDAC3, SLC20A2, C8orf40, PLEC, MIR661, SLC34A3, TUBB4B, PHEX, PHEX-AS1, ZNF645, CLCN5, SNX12, TCEAL6, TCEAL7, LOC158696, FGF13

Table IV. Primer sequences used to amplify the 22 PHEX exons.

\begin{tabular}{|c|c|c|}
\hline Exon & Forward primer & Reverse primer \\
\hline 1 & AGGGACTTTGCTgagggagag & CCACTCGAAGCCACTTAcacc \\
\hline 2 & TGGGTTTTGGAATACCGTGTC & AAGAGAGGccattcagccttc \\
\hline 3 & caaGGCTTGGAAACTGGTTGA & TTATGTTGAGATCTGGGAGTCCA \\
\hline 4 & GGCACCATATGTGGGTGGATA & GTTTGCCCTGCTGACTTTGTC \\
\hline 5 & CACATTGAAGCGTGGATCGTA & CGGGAGAAGGGaatattctgg \\
\hline 6 & GCTCTGCCCAATCAtgttacc & GCAGCCTGGTAAGGCACATAG \\
\hline 7 & GGGTGCCTGGTATTGCATAAT & CCAATGGGCAATGACACAAA \\
\hline 8 & ACCACACCaaagccttgaaaa & GAGCCAATGCCAACAATTACC \\
\hline 9 & GGATGGCAATGAtcaggagtt & GACAGTGCTTTTGGCCAGTTC \\
\hline 10 & atgttcactctgagggctgga & GGCTACAAACTCCCCCTGTCT \\
\hline 11 & cagccatgggttttatccaaa & cccactcccetgGAAAACTac \\
\hline 12 & agtgttGCCAGAGCATGGAGT & aggaaaggccGAAttacaagg \\
\hline 13 & TCGATTCAGTCACCTTCTcса & GAAAGGCACAAGGCCAGtaaa \\
\hline 14 & tgactGATGCAGCTTCTCTGC & ATGCTAGAAATGGGGGACCTG \\
\hline 15 & gcagggaCAGCCCtttagatt & GCcacttttgggggaaataag \\
\hline 16 & GTGCAAAATGGtttccctgaa & GTCCAGCCATACACCCTGGTa \\
\hline 17 & AAGCAGTTTATCTTGGCtttcca & CAAGCCATCACAGCAAGACAC \\
\hline 18 & CTGCTTTTTGAAGGCTTGTCg & ATGCCTGGTTAAGGGATGacc \\
\hline 19 & ttGATGCCTCTTGCTGAATGA & aaatgaacctagCCCCAAGGA \\
\hline 20 & TGGTAAGCAACAGGACATGGA & AGGGCTGCTAACccatttgat \\
\hline 21 & ttcctgggcacatataCGattc & ttttggctgcaaaatggaaat \\
\hline 22 & cagaACCTGTTGATGTGcaaga & GCCAACACCCTAAAATGGaca \\
\hline
\end{tabular}

PHEX, phosphate-regulating gene with homologies to endopeptidases on the $\mathrm{X}$ chromosome.

annotation databases downloaded from the UCSC Genome Browser. Variants were further filtered using the following criteria: SNP and indel quality score $\geq 100$; and genotype score as a Phred-scaled confidence at the true genotype $\geq 40.0$. The number of read depths for mapping the read quality $\geq 17$ was calculated as $\geq 20$. Then, functional variants were selected by examining whether they were within the protein coding sequence or splicing sites as defined by RefSeq gene models.

Selection of candidate disease-related gene loci. Selection of candidate disease-related gene loci was performed by Shanghai BioGenius Co. Ltd. (Shanghai, China). To select the 
candidate disease-related gene loci, association analysis and genetic model- and frequency-based filtering strategies were performed. First, PLINK analysis was applied to conduct targeted exome-based association analysis using the following five statistical models: Allelic test, genotype assumptions test, additive assumptions test, dominant assumptions test, and recessive assumptions test. In specific, the basic association test for a disease trait is based on comparing the allele frequencies between cases and controls (asymptotic and empirical P-values are available). The Cochran-Armitage trend test, Fisher's exact test, and different genetic models (dominant, recessive and general) were implemented using PLINK. Of the five results, the minimum P-value was selected for each SNP as the most significant loci in the final results. Next, the candidate disease-related loci were selected based on the genetic model. The dominant patients should have heterozygous mutation genotypes when the controls are all wild-type genotypes. By contrast, the recessive patients should have homozygote mutation genotypes when the controls are all wild-type genotypes.

Confirmation of PHEXand DMP1 gene mutations. According to the next-generation sequencing results, 22 coding exons were sequenced from the PHEX gene [using GenBank accessions to screen for mutations using 22 primer pairs (Table IV) in patients 1-7]. The primers were designed using Primer3 (http://frodo.wi.mit.edu/cgi-bin/primer3/primer3 www.cgi). The PHEX gene coding region was amplified by PCR. Direct nucleotide sequencing was performed using the Big-Dye Terminator Cycle Sequencing Ready Reaction kit version 3.1 (Applied Biosystems; Thermo Fisher Scientific, Inc.) and analysed using an ABI 3130 automatic sequencer (Applied Biosystems; Thermo Fisher Scientific, Inc.) following standard methods.

One pair of primers was designed to detect known mutations in the $D M P 1$ gene in patient 8 . The following $D M P 1$ primers were designed using Primer3: P7F, GCAGGGAAC TTGACAATGAGG; and P7R, GGGTTATCTCCCCTGGAC TCA. Direct nucleotide sequencing was performed using the Big-Dye Terminator Cycle Sequencing Ready Reaction kit version 3.1 and analysed using an ABI 3130 automatic sequencer, following standard methods.

Following confirmation among these 8 patients, Sanger confirmation was completed on their family members.

Fourteen variants via Sanger confirmation. A total of 14 mutation sites were sequenced from 12 genes from patients according to the targeted exome sequencing data. The primers were designed using Primer3 (Table V). Direct nucleotide sequencing was performed using the Big-Dye Terminator Cycle Sequencing Ready Reaction kit version 3.1 and analysed using an ABI 3130 automatic sequencer, following standard methods.

Measuring circulating intact FGF23 levels. Serum FGF23 levels were measured using an intact human FGF23 ELISA kit obtained from Kainos Laboratories Inc. (Tokyo, Japan; cat. no. CY-4000). The detectable concentration ranged from 3 to $800 \mathrm{pg} / \mathrm{ml}$. This assay was validated in our previous study (19).

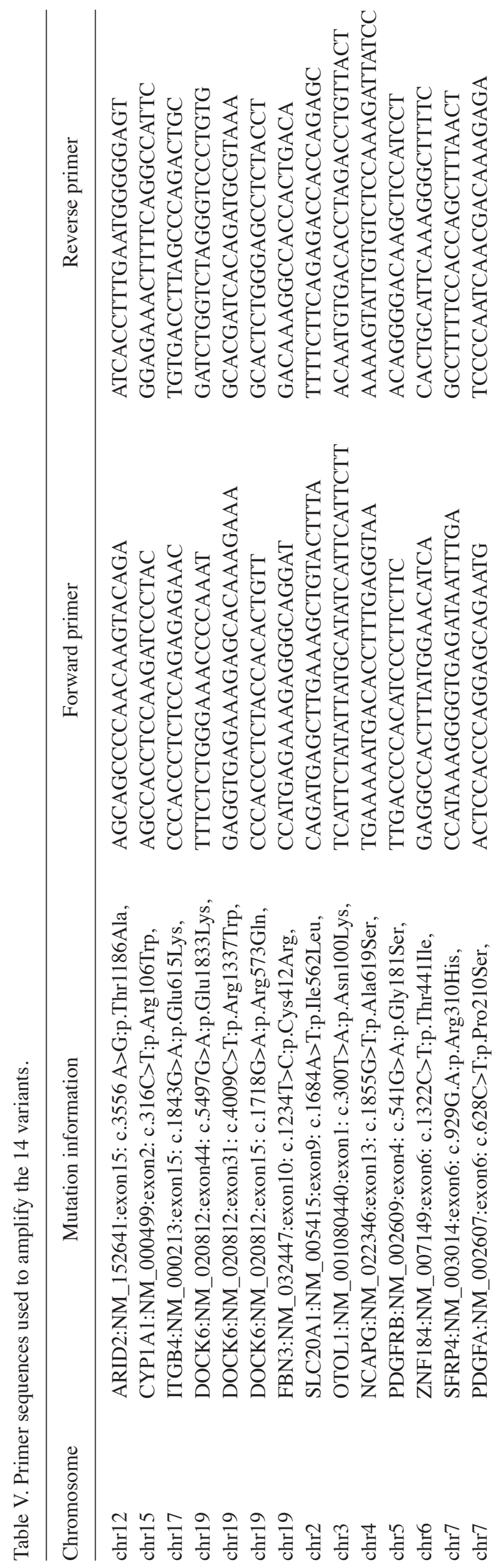




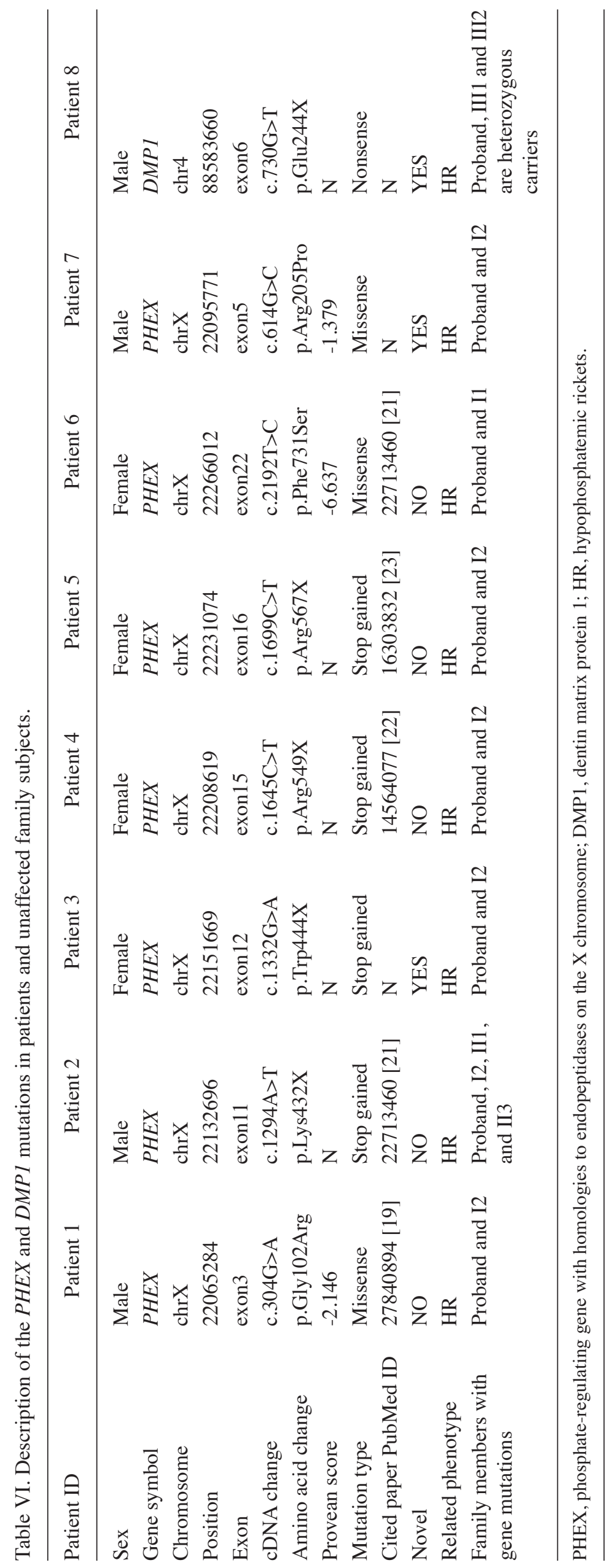


Statistical analysis. Normally distributed variables are presented as the mean \pm standard deviation, and non-normally distributed variables as the median (25 and 75 th percentiles). $\mathrm{P}<0.05$ was considered to indicate a statistically significant differences. Statistical analyses were performed using SPSS 13.0 software (SPSS Inc., Chicago, IL, USA).

\section{Results}

Variants in known reported disease-related genes discovered in the present cohort. In the current targeted next-generation sequencing (NGS) study, the mean read depth of the target regions was greater than 100x for all of the subjects (data not shown).

A total of 86 patients with biochemically and clinically confirmed hypophosphatemic rickets/osteomalacia and 100 unrelated healthy controls were included. Following selection of candidate disease-related gene loci, seven different PHEX (located on the $\mathrm{X}$ chromosome at Xp22.1) mutations were identified in seven unrelated patients, two of which were novel according to the PHEX Locus Database (http://www.phexdb. mcgill.ca/; accessed January 2018) (20). Table VI contains a complete description of the identified mutations (21-23). The seven PHEX mutations included three missense and four stop-gain mutations. In addition, one novel nonsense mutation (c.730G > T, p.Glu244X) was identified in exon 6 of DMPI (MIM 600980) that was located at 4q21 (4) in an 18-year-old boy (Table VI); this result was confirmed according to HGMD (http://www.hgmd.org/) and a reference review. Autosomal recessive hypophosphatemic rickets (MIM 241520) is defined by mutations in DMP1. DMP1 belongs to a group of genes encoding a class of bone and tooth noncollagenous matrix proteins known as SIBLING (small integrin-binding ligand, $\mathrm{N}$-linked glycoproteins) (24). In family 8, the proband (IV-1), an 18-year-old boy, was born full-term from a consanguineous marriage. He was the only patient in the family. At 18 months of age, the patient complained of painful legs and displayed varus knee. His height is $145 \mathrm{~cm}$ and weight is $45 \mathrm{~kg}$. Additionally, he had poor dental development, with normal vision and hearing. His serum ALP, Ca, and P levels were 741U/1, 2.46 and $0.76 \mathrm{mmol} / 1$, respectively. The radiograph shows his lower extremity genu varus deformities (Fig. 2). The clinical features of the affected individuals in these eight families are listed in Table VII.

Pathogenic mutation prediction and Sanger confirmation. After filtering out the non-functional variants, the remaining nonsynonymous or frameshift single nucleotide variants (SNVs) were assessed as the damaging effect. Mutation deleterious predictions for these mutations were performed by using sorting intolerant from tolerant (SIFT) algorithm, polymorphism phenotyping v2 (PolyPhen2) algorithm and MutationTaster. Then, the 14 heterozygous variants from 12 genes were determined. According to the Exome Aggregation Consortium database, 4 variants were novel, and the other 10 variants were rare or vary rare variants (Table VIII). These genes included secreted frizzled related protein 4 (SFRP49), fibrillin 3 (FBN3), cytochrome P450 family 1 subfamily A member 1 (CYPIAl), AT-rich interaction domain 2 (ARID2), otolin 1 (OTOL1), dedicator of

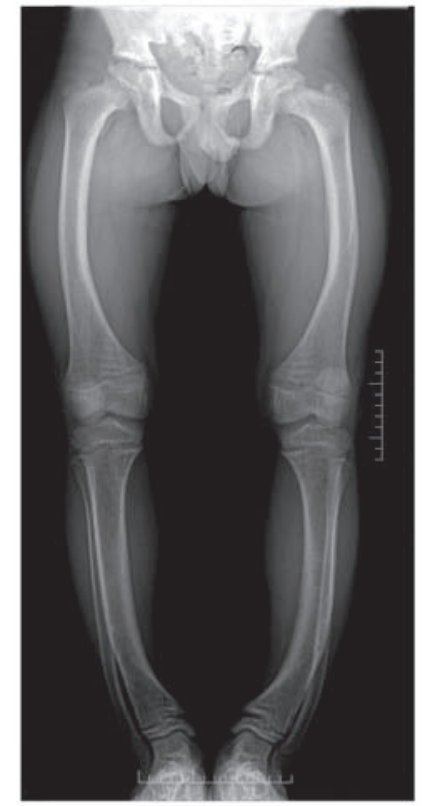

Figure 2. Radiology result for the patient with DMP1 mutation. The radiograph shows lower extremity genu varus deformities.

cytokinesis 6 (DOCK6), platelet-derived growth factor receptor $\beta$ polypeptide $(P D G F R B)$, solute carrier family 20 member 1 (SLC2OA1), integrin subunit $\beta 4$ (ITGB4), non-SMC condensin I complex subunit G (NCAPG), platelet-derived growth factor subunit A (PDGFA), and zinc finger protein 184 (ZNF184). The variants were confirmed by Sanger sequencing. These 14 patients did not have family histories, and two of the patients were younger than 18 years old. They all had typical clinical manifestations of hypophosphatemic rickets/osteomalacia. Enrichment analysis for the 14 variants was conducted using the Database for Annotation, Visualization and Integrated Discovery tool (Fig. 3). The amino acids at the mutation sites are highly conserved in the 8 different species. Variants found in the present cohort in known reported disease-related genes (PHEX and DMP1) are further discussed in Table VI.

Confirmation of PHEX and DMPI gene mutations. The Sanger sequencing results for the PHEX and DMPI gene mutation sites were consistent with the targeted NGS sequencing results (Fig. 4). The c.304G $>$ A mutation of the PHEX gene was found in patient 1 and his mother (I2). The c.1294A $>$ T mutation of the PHEX gene was found in patient 2 and three affected individuals (I2, II1, and II3). The c.1332G $>$ A mutation of the PHEX gene was found in patient 3 and her mother (I2). The c. $1645 \mathrm{C}>\mathrm{T}$ mutation of the $P H E X$ gene was found in patient 4 and her mother (I2). The c.1699C $>$ T mutation of the $P H E X$ gene was found in patient 5 and her mother (I2). The c.2192T $>C$ mutation of the $P H E X$ gene was found in patient 6 and her father (I1). The c.614G $>$ C mutation of the $P H E X$ gene was found in patient 7 and his mother (I2). The homozygous c.730G $>$ T mutation from the $D M P 1$ gene was found in patient 8 , although both of his parents were heterozygous carriers. In family 8 , although the family chart included 4 generations, only the proband and his parents were tested, because DNA samples 
Table VII. Clinical presentation and laboratory results of the patients with PHEX mutation and DMP1 mutation in the present study.

\begin{tabular}{|c|c|c|c|c|c|c|c|c|c|c|}
\hline Family no. & Patient & Sex & $\begin{array}{c}\text { Age } \\
\text { (years) }\end{array}$ & Clinical characteristics & $\begin{array}{l}\text { Calcium } \\
(\mathrm{mmol} / \mathrm{l})\end{array}$ & $\begin{array}{l}\text { Phosphorus } \\
\text { (mmol/l) }\end{array}$ & $\begin{array}{l}\text { ALP } \\
(u / 1)\end{array}$ & $\begin{array}{c}\text { PTH } \\
(\mathrm{pg} / \mathrm{ml})\end{array}$ & $\begin{array}{l}25(\mathrm{OH}) \mathrm{D} \\
(\mathrm{ng} / \mathrm{ml})\end{array}$ & $\begin{array}{l}\text { FGF23 } \\
(\mathrm{pg} / \mathrm{ml})\end{array}$ \\
\hline 1 & $\mathrm{I}-2$ & $\mathrm{~F}$ & 48 & Short stature & 2.28 & 0.68 & 96 & 52.3 & 17.6 & 30.25 \\
\hline 1 & II-1 & M & 21 & Genu varum, short stature & 2.32 & 0.43 & 109 & 68.3 & 15.4 & 48.23 \\
\hline 2 & $\mathrm{I}-2$ & $\mathrm{~F}$ & 79 & Genu varum, short stature & 2.37 & 0.81 & 84 & $\mathrm{~N}$ & $\mathrm{~N}$ & $\mathrm{~N}$ \\
\hline 2 & II-1 & M & 58 & $\begin{array}{l}\text { Genu varum, short stature, } \\
\text { teeth falling out }\end{array}$ & 2.35 & 0.73 & 165 & 72.2 & 15.6 & 39.62 \\
\hline 2 & II-3 & $\mathrm{F}$ & 56 & Genu varum, short stature & 2.34 & 0.82 & 88 & 42.5 & 25.2 & 25.08 \\
\hline 2 & III-1 & M & 30 & $\begin{array}{l}\text { Genu varum, short stature, } \\
\text { teeth falling out }\end{array}$ & 2.32 & 0.46 & 108 & 59.2 & 21.3 & 38.72 \\
\hline 3 & $\mathrm{I}-2$ & $\mathrm{~F}$ & 73 & Short stature, teeth falling out & 2.29 & 0.53 & 180 & 93.6 & 10.5 & 52.17 \\
\hline 3 & II-1 & $\mathrm{F}$ & 47 & Short stature, teeth falling out & 2.24 & 0.46 & 378 & 109.7 & 18.9 & 30.29 \\
\hline 4 & $\mathrm{I}-2$ & $\mathrm{~F}$ & 62 & Genu varum, short stature & 2.34 & 0.48 & 128 & 58.9 & 34.3 & 16.84 \\
\hline 4 & II-1 & $\mathrm{F}$ & 31 & Genu varum, short stature & 2.18 & 0.50 & 122 & 48.4 & 11.3 & 12.05 \\
\hline 5 & $\mathrm{I}-2$ & $\mathrm{~F}$ & 52 & Short stature & 2.20 & 0.65 & 222 & 127.1 & 8.8 & 62.36 \\
\hline 5 & II-1 & $\mathrm{F}$ & 28 & Short stature, hard to walk & 2.04 & 0.45 & 173 & 147.4 & 19.4 & 19.25 \\
\hline 6 & $\mathrm{I}-1$ & M & 48 & Genu varum, short stature & 2.41 & 0.76 & 124 & 47.1 & 14.5 & 44.23 \\
\hline 6 & II-2 & $\mathrm{F}$ & 21 & $\begin{array}{l}\text { Genu varum, short stature, } \\
\text { bone pain }\end{array}$ & 2.40 & 0.62 & 89 & 52.1 & 17.1 & 21.56 \\
\hline 7 & $\mathrm{I}-2$ & $\mathrm{~F}$ & 33 & Genu varum, short stature & 2.13 & 0.43 & 147 & 48.4 & 10.2 & 50.72 \\
\hline 7 & II-1 & M & 9 & $\begin{array}{l}\text { Genu varum, short stature, } \\
\text { growth retardation }\end{array}$ & 2.38 & 0.33 & 697 & 211.1 & 17.3 & 110.23 \\
\hline 8 & IV-1 & M & 18 & $\begin{array}{l}\text { Genu varum, short stature, } \\
\text { growth retardation }\end{array}$ & 2.46 & 0.76 & 741 & 69.1 & 19.2 & 91.56 \\
\hline
\end{tabular}

Abnormal data are denoted in bold font. Normal ranges are: phosphate, 0.8-1.6 mmol/l; calcium, 2.08-2.60 mmol/1; ALP, 15-112 u/l; PTH, 15-65 m/l; and 25(OH)D, $\geq 20 \mathrm{ng} / \mathrm{ml}$. F, female; M, male; N, not tested; ALP, alkaline phosphatase; PTH, parathyroid hormone; 25(OH)D, 25-OH vitamin D; FGF23, fibroblast growth factor 23 .

could not be obtained from the grandparents (Fig. 1). No mutations were detected in the phenotype-normal family members of the other 7 families or the 100 ethnic-matched control individuals. The c.304G $>$ A mutation, c.2192T $>C$ mutation and c.614G $>\mathrm{C}$ mutation of the $P H E X$ gene were predicted to most likely be damaging (PolyPhen scores of $-2.146,-6.637$, and -1.379 , respectively). The amino acids at these mutation sites are highly conserved in the 8 different species.

Serum FGF23 levels. Serum FGF23 levels were not normally distributed in either the normal controls or the hypophosphatemic rickets/osteomalacia patients. The median value for intact serum FGF23 levels in the 100 healthy individuals was $39.83 \mathrm{pg} / \mathrm{ml}$, and the 25 and 75 th percentiles were 25.82 and $93.69 \mathrm{pg} / \mathrm{ml}$, respectively. For the patients with hypophosphatemic rickets/osteomalacia, as presented in Table II, the median value for intact serum FGF23 levels was $38.29 \mathrm{pg} / \mathrm{ml}$, and the 25 and 75th percentiles were 22.09 and $89.55 \mathrm{pg} / \mathrm{ml}$, respectively. The serum FGF23 levels exhibited a wide variation in the patients with hypophosphatemic rickets/osteomalacia, and no significant differences were found compared with those of the normal controls.

\section{Discussion}

All of the selected patients displayed typical hypophosphatemic rickets/osteomalacia phenotypes, and seven patients had family histories of the disease. Laboratory analysis of the patients' sera revealed low phosphorus levels, normal calcium levels and elevated ALP levels. ${ }^{18} \mathrm{~F}-\mathrm{FDG}$ PET/CT and 99 mTc-OCT scintigraphy were performed on 10 and 20 of the patients, respectively. In addition, the FGF23 levels were normal. Currently, there is no evidence that these subjects have the potential to have TIO.

In the present study, a diagnostic panel was described for the molecular diagnosis of hypophosphatemic rickets/osteomalacia. Followed by massively parallel sequencing, the target genes were enriched through a solution-based oligonucleotide probe hybridization capture approach. In total, 196 genes were simultaneously sequenced with deep coverage. Using this method, mutations in common genes (PHEX and DMPI) were identified in eight subjects with hypophosphatemic rickets/osteomalacia without prior molecular testing.

PHEX gene mutations in the Chinese population have been repeatedly reported; for example, we have previously reported 


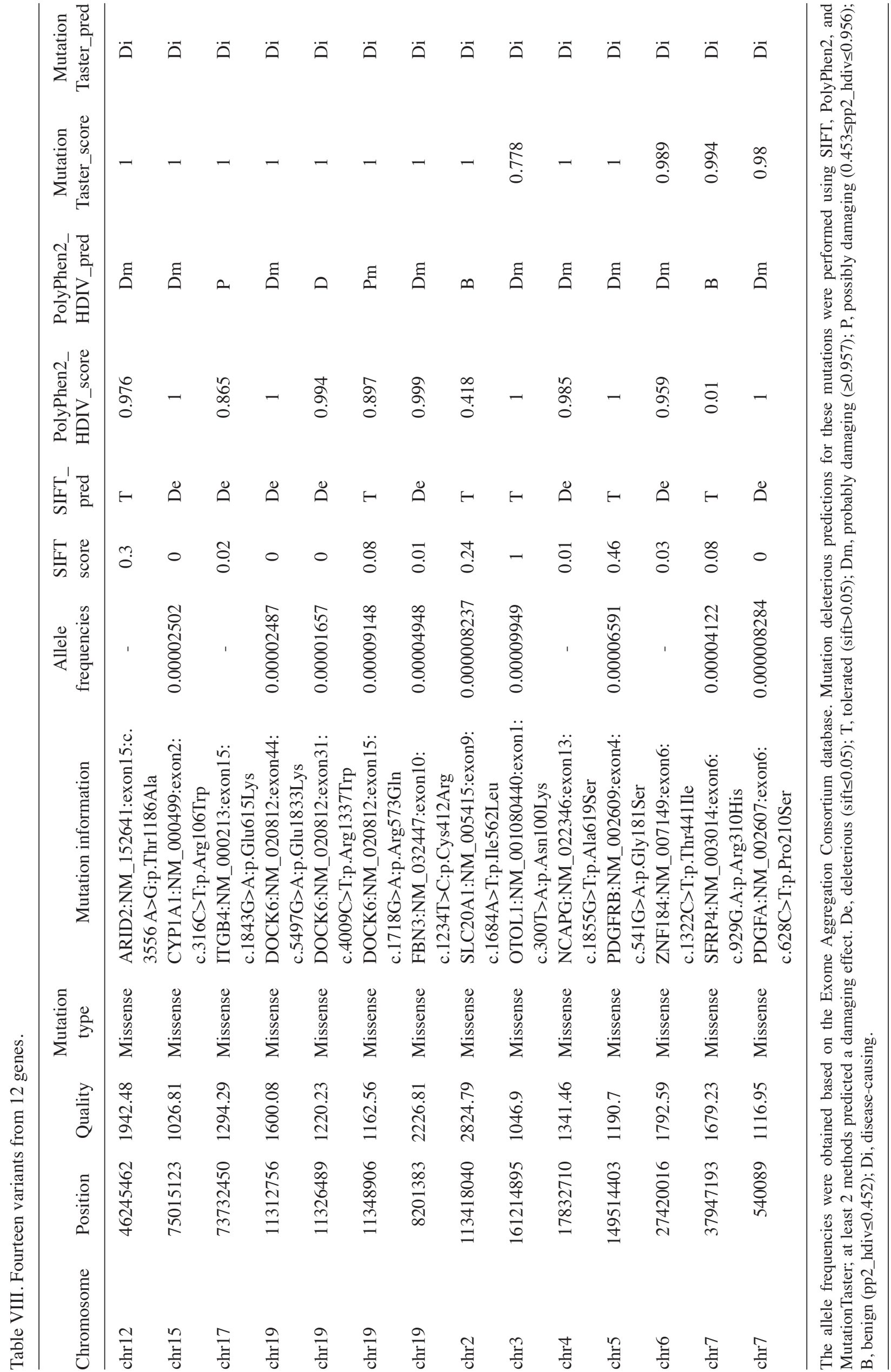




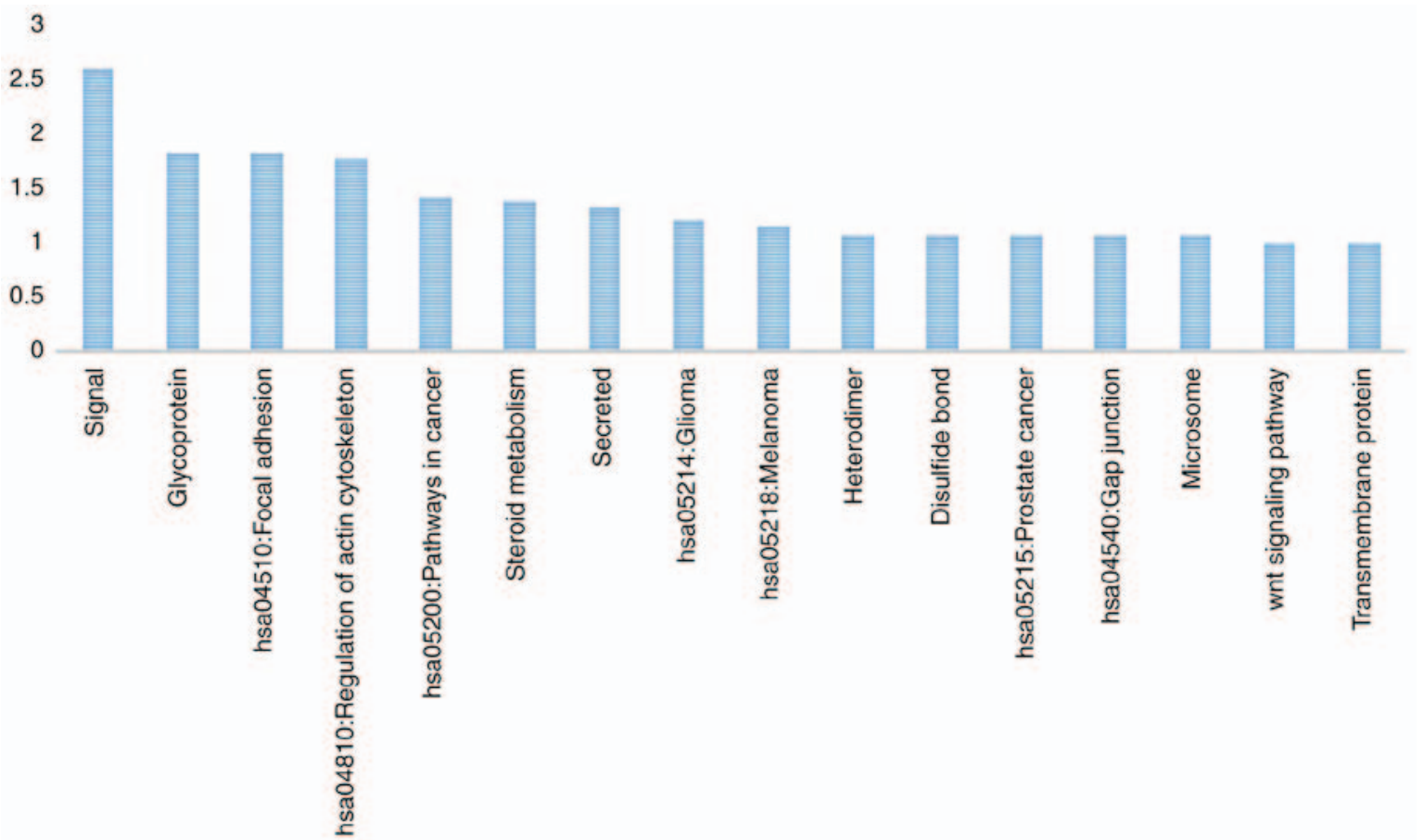

Figure 3. Enrichment analysis was conducted for the variants using the Database for Annotation, Visualization and Integrated Discovery tool.

A
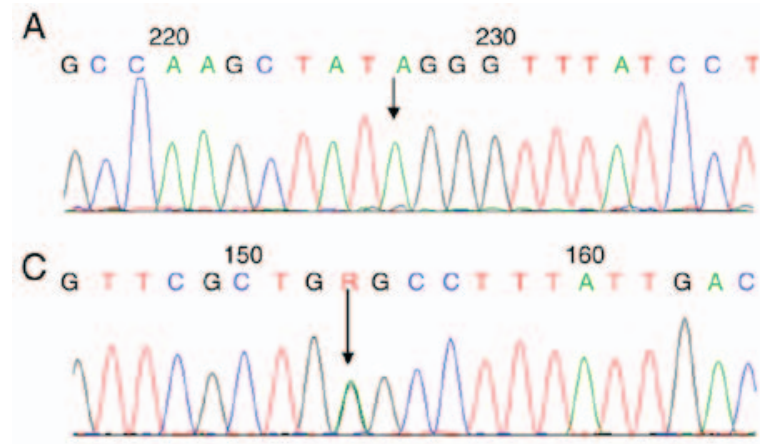

E 230

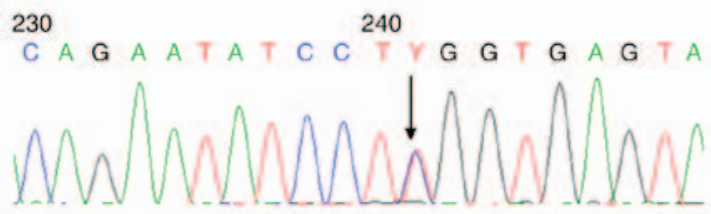

G

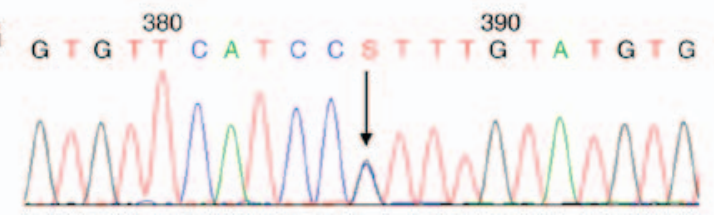

B
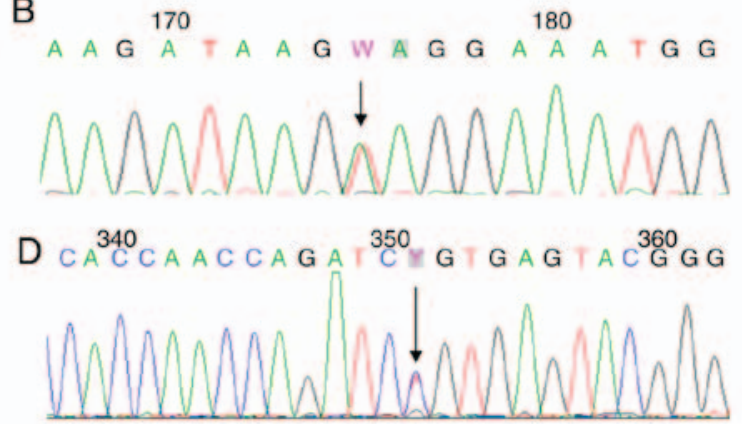

F

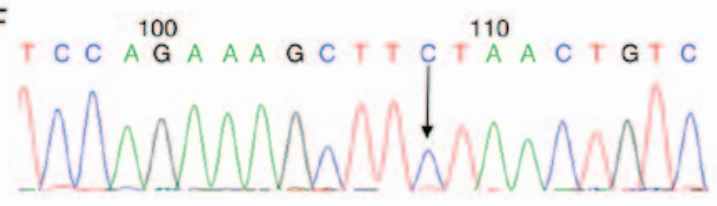

$\mathrm{H}$

200

210

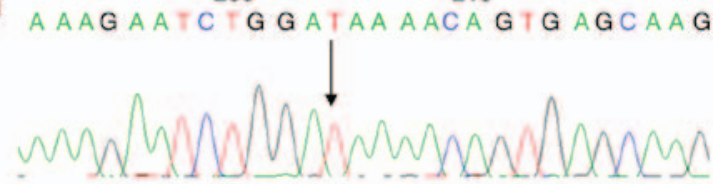

Figure 4. Mutational analysis. (A) c.304G $>$ A mutation in exon 3 of the $P H E X$ gene. (B) c.1294A $>$ T mutation in exon 11 of the $P H E X$ gene. (C) c.1332G $>$ A mutation in exon 12 of the PHEX gene. (D) c.1645C $>$ T mutation in exon 15 of the $P H E X$ gene. (E) c.1699C $>$ T mutation in exon 16 of the $P H E X$ gene. (F) c.2192T $>\mathrm{C}$ mutation in exon 22 of the PHEX gene. (G) c.614G>C mutation in exon 5 of the PHEX gene. (H) c.730G $>\mathrm{T}$ mutation in exon6 of the DMP1 gene. PHEX, phosphate-regulating gene with homologies to endopeptidases on the X chromosome; DMP1, dentin matrix protein 1.

several cases $(19,21,25)$. By contrast, $D M P 1$ gene mutations have not been reported in the Chinese population, although one DMPI mutation has been reported in a Japanese subject. In 2010, Koshida et al (26) identified a novel homozygous nonsense mutation $(98 \mathrm{G}>\mathrm{A}, \mathrm{W} 33 \mathrm{X})$ in the $D M P 1$ gene from a Japanese family with autosomal recessive hypophosphatemic rickets.
All of the patients included in the present study had typical osteomalacia or rickets symptoms and low serum phosphorus levels. Fourteen heterozygous variants were identified, four of which were novel. These variants are in the following 12 genes: SFRP4, FBN3, CYP1A1, ARID2, OTOL1, DOCK6, PDGFRB, SLC20A1, ITGB4, NCAPG, PDGFA, and ZNF184. The amino 
acids at these mutation sites are highly conserved in the eight different species. Among the 14 variants, two of the genes, $S L C 20 A 1$ and PDGFRB, are of predominant interest.

SLC20A1/PiT1 (OMIM 137570), a member of the SLC20 family of sodium-coupled Pi transporters, has been considered a putative Pi transporter in bone for a long time. Notably, SLC20A1 has been proved to be upregulated in osteoblastic cells at the initial stage of mineralization. However, the physiological role of $S L C 20 A 1$ in bone remains unclear. The discrete expression of SLC20A1 in apoptotic hypertrophic chondrocytes late in development is consistent with an apoptosis-related and Pi transport-independent function of $S L C 20 A 1$ in bone (27). Low levels of SLC20A1 could modulate the destiny of hypertrophic chondrocytes at the mineralization front. In the present study, a heterozygous c.1684A $>$ T mutation (p.I562L) was identified in a patient in the SLC20Al gene. The patient was a 13-year-old boy whose clinical features consisted of an abnormal gait, delayed dentition, spinal double concave, and genu extroversion with an ' $\mathrm{X}$ ' appearance. The PDGFRB gene (OMIM 190040) encodes a cell surface tyrosine kinase receptor for members of the platelet-derived growth factor family. The PDGFB-PDGFRB pathway seems to be related to phosphate-induced calcifications in vascular smooth muscle cells by regulating the expression of the phosphate transporter SLC20A1 (28). Currently, it is recognized that calcification of vascular smooth muscle cells occurs via a process similar to that of normal bone formation. In the present study, a heterozygous c.541G $>$ A (p.G181S) mutation was identified in a patient in the PDGFRB gene. The patient was a 54-year-old woman who had a short stature, mild genu varum, and bilateral femoral pseudofractures. Further experiments to confirm these two heterozygous mutations among more hypophosphatemic rickets/osteomalacia patients will be performed in the future. In addition, functional studies investigating these mutations should be performed to elucidate the complex relationships between genotype and phenotype.

Following enrichment analysis, a total of 16 pathways reached a nominal $\mathrm{P}<0.05$ (Fig. 3). The majority of the pathways were not related to bone metabolism. The WNT-signalling pathway is of the most interest. Wnt signals are known to affect the differentiation of osteoblasts. Osteoblasts are the predominant functional cells of bone formation and are responsible for the synthesis, secretion and mineralization of bone matrix. Hypophosphatemic rickets/osteomalacia is a disease characterized by impaired mineralization of bone matrix. Therefore, it can be hypothesized that the Wnt signalling pathway may be related to the pathogenesis of hypophosphatemic rickets/osteomalacia. Its mechanism remains to be revealed by future research.

The present study had several limitations that should be acknowledged. First, when selecting the target sites, the consideration was not comprehensive enough, thus omitting some important genes, such as $\alpha$-Klotho, the co-receptor for FGF23. Overexpression of $\alpha$-Klotho, both in humans and mice, has been demonstrated to lead to hypophosphatemia (29). Second, our sample size was not very large, with 86 hypophosphatemic rickets/osteomalacia patients included in the present study. In addition, DNA samples from most unaffected family members were not collected, and thus parental testing of the 14 heterozygous variants was not performed. Third, except for common genes (PHEX and DMP1), other homozygous mutations were not discovered in the present study. Hence, the sample size should be enlarged and complete family member data should be collected in the future. Furthermore, with the quick development of NGS, the cost will be further reduced, and whole exome sequencing technology may be directly used to explore the pathogenic genes in these patients.

In conclusion, the present study extended the spectrum of mutations in the PHEX and DMPI genes that cause hypophosphatemic rickets/osteomalacia. Additionally, the results suggested that heterozygous mutations in other candidate genes might also contribute to this disease. These assumptions must be validated by further sequencing projects and functional tests. However, the current study demonstrated that the present diagnostic platform can successfully be used in the clinical setting for the accurate and rapid molecular diagnosis of patients with hypophosphatemic rickets/osteomalacia. Future directions would include the incorporation of additional genes into the existing panel and the design of new panels that target other causes of skeletal dysplasia.

\section{Acknowledgements}

We are grateful to the patients and their families for participating in this study. We thank the Center for Genetic \& Genomic Analysis, Genesky Biotechnologies Inc. (Shanghai, China) and the Shanghai BioGenius Co. Ltd. (Shanghai, China) for technical assistance.

\section{Funding}

This study was supported by the National Basic Research Program of China (grant no. 2014CB942903), the National Natural Science Foundation of China (grant nos. 81200646, 81370978 and 81400852), Science and Technology Commission of Shanghai Municipality (grant no. 14JC1405000) and Shanghai Municipal Planning Commission of science and Research Fund (grant no. 20144Y0127).

\section{Availability of data and materials}

The analyzed datasets generated during the study are available from the corresponding author on reasonable request.

\section{Authors' contributions}

JG participated in the recruitment of patients, acquisition of data, analysis and interpretation of data, and drafting of the manuscript. $\mathrm{CW}, \mathrm{HZ}, \mathrm{HY}$ and $\mathrm{WH}$ participated in the recruitment of patients. WF and $\mathrm{JH}$ performed the molecular genetic studies and participated in the sequence alignment. ZZ conceived the study, participated in its design, and gave final approval for the version. All authors read and approved the final manuscript.

\section{Ethics approval and consent to participate}

The present study was approved by the Ethics Committee of the Shanghai Jiao Tong University-Affiliated Sixth People's Hospital [nos. 2013-87 and 2014-KY-001(k)]. Signed 
informed consent was obtained from all the subjects prior to participation in the study.

\section{Patient consent for publication}

Consent to publish was obtained from all the participants.

\section{Competing interests}

The authors declare that they have no competing interests.

\section{References}

1. Rajah J, Thandrayen K and Pettifor JM: Clinical practice: Diagnostic approach to the rachitic child. Eur J Pediatr 170 1089-1096, 2011

2. A gene (PEX) with homologies to endopeptidases is mutated in patients with X-linked hypophosphatemic rickets. The HYP Consortium. Nat Genet 11: 130-136, 1995.

3. ADHR Consortium: Autosomal dominant hypophosphataemic rickets is associated with mutations in FGF23. Nat Genet 26: 345-348, 2000.

4. Lorenz-Depiereux B, Bastepe M, Benet-Pagès A, Amyere M, Wagenstaller J, Müller-Barth U, Badenhoop K, Kaiser SM, Rittmaster RS, Shlossberg AH, et al: DMP1 mutations in autosomal recessive hypophosphatemia implicate a bone matrix protein in the regulation of phosphate homeostasis. Nat Genet 38 1248-1250, 2006.

5. Feng JQ, Ward LM, Liu S, Lu Y, Xie Y, Yuan B, Yu X, Rauch F Davis SI, Zhang S, et al: Loss of DMP1 causes rickets and osteomalacia and identifies a role for osteocytes in mineral metabolism. Nat Genet 38: 1310-1315, 2006.

6. Levy-Litan V,HershkovitzE,Avizov L, LeventhalN, Bercovich D, Chalifa-Caspi V, Manor E, Buriakovsky S, Hadad Y, Goding J and Parvari R: Autosomal-recessive hypophosphatemic rickets is associated with an inactivation mutation in the ENPP1 gene. Am J Hum Genet 86: 273-278, 2010.

7. Lorenz-Depiereux B, Schnabel D, Tiosano D, Häusler G and Strom TM: Loss-of-function ENPP1 mutations cause both generalized arterial calcification of infancy and autosomal-recessive hypophosphatemic rickets. Am J Hum Genet 86: 267-272, 2010.

8. Rafaelsen SH, Raeder H, Fagerheim AK, Knappskog P, Carpenter TO, Johansson S and Bjerknes R: Exome sequencing reveals FAM20c mutations associated with fibroblast growth factor 23-related hypophosphatemia, dental anomalies, and ectopic calcification. J Bone Miner Res 28: 1378-1385, 2013.

9. Bergwitz C, Roslin NM, Tieder M, Loredo-Osti JC, Bastepe M, Abu-Zahra H, Frappier D, Burkett K, Carpenter TO, Anderson D, et al: SLC34A3 mutations in patients with hereditary hypophosphatemic rickets with hypercalciuria predict a key role for the sodium-phosphate cotransporter NaPi-IIc in maintaining phosphate homeostasis. Am J Hum Genet 78: 179-192, 2006.

10. Lorenz-Depiereux B, Benet-Pages A, Eckstein G, Tenenbaum-Rakover Y, Wagenstaller J, Tiosano D, Gershoni-Baruch R, Albers N, Lichtner P, Schnabel D, et al: Hereditary hypophosphatemic rickets with hypercalciuria is caused by mutations in the sodium-phosphate cotransporter gene SLC34A3. Am J Hum Genet 78: 193-201, 2006.

11. Brakemeier S, Si H, Gollasch M, Höffler D, Buhl M, Köhler R, Hoyer J and Eichler I: Dent's disease: Identification of a novel mutation in the renal chloride channel CLCN5. Clin Nephrol 62: 387-390, 2004.

12. Cho HY, Lee BH, Choi HJ, Ha IS, Choi Y and Cheong HI: Renal manifestations of dent disease and lowe syndrome. Pediatr Nephrol 23: 243-249, 2008.
13. Dhir G, Li D, Hakonarson H and Levine MA: Late-onset hereditary hypophosphatemic rickets with hypercalciuria (HHRH) due to mutation of SLC34A3/NPT2c. Bone 97: 15-19, 2017.

14. Goljanek-Whysall K, Tridimas A, McCormick R, Russell NJ, Sloman M, Sorani A, Fraser WD and Hannan FM: Identification of a novel loss-of-function PHEX mutation, Ala720Ser, in a sporadic case of adult-onset hypophosphatemic osteomalacia. Bone 106: 30-34, 2018.

15. Harismendy O, Ng PC, Strausberg RL, Wang X, Stockwell TB, Beeson KY, Schork NJ, Murray SS, Topol EJ, Levy S and Frazer KA: Evaluation of next generation sequencing platforms for population targeted sequencing studies. Genome Biol 10: R32, 2009.

16. Okou DT, Steinberg KM, Middle C, Cutler DJ, Albert TJ and Zwick ME: Microarray-based genomic selection for high-throughput resequencing. Nat Methods 4: 907-909, 2007.

17. Ng SB, Turner EH, Robertson PD, Flygare SD, Bigham AW, Lee C, Shaffer T, Wong M, Bhattacharjee A, Eichler EE, et al: Targeted capture and massively parallel sequencing of 12 human exomes. Nature 461: 272-276, 2009.

18. Wang C, Zhang H, He JW, Gu JM, Hu WW, Hu YQ, Li M, Liu YJ, Fu WZ, Yue H, et al: The virulence gene and clinical phenotypes of osteopetrosis in the Chinese population: Six novel mutations of the CLCN7 gene in twelve osteopetrosis families. J Bone Miner Metab 30: 338-348, 2012.

19. Li SS, Gu JM, Yu WJ, He JW, Fu WZ and Zhang ZL: Seven novel and six de novo PHEX gene mutations in patients with hypophosphatemic rickets. Int J Mol Med 38: 1703-1714, 2016.

20. Grieff M, Mumm S, Waeltz P, Mazzarella R, Whyte MP, Thakker RV and Schlessinger D: Expression and cloning of the human X-linked hypophosphatemia gene cDNA. Biochem Biophys Res Commun 231: 635-639, 1997.

21. Kang QL, Xu J, Zhang Z, He JW, Lu LS, Fu WZ and Zhang ZL: Three novel PHEX gene mutations in four Chinese families with X-linked dominant hypophosphatemic rickets. Biochem Biophys Res Commun 423: 793-798, 2012.

22. Popowska E, Pronicka E, Sułek A, Jurkiewicz D, Rowe P, Rowinska E and Krajewska-Walasek M: X-linked hypophosphatemia in Polish patients. 1. Mutations in the PHEX gene. J Appl Genet 41: 293-302, 2000.

23. Goji K, Ozaki K, Sadewa AH, Nishio H and Matsuo M: Somatic and germline mosaicism for a mutation of the PHEX gene can lead to genetic transmission of X-linked hypophosphatemic rickets that mimics an autosomal dominant trait. J Clin Endocrinol Metab 91: 365-370, 2006.

24. Fisher LW and Fedarko NS: Six genes expressed in bones and teeth encode the current members of the SIBLING family of proteins. Connect Tissue Res 44 (Suppl 1): S33-S40, 2003.

25. Yue H, Yu JB, He JW, Zhang Z, Fu WZ, Zhang H, Wang C, $\mathrm{Hu}$ WW, Gu JM, Hu YQ, et al: Identification of two novel mutations in the PHEX gene in Chinese patients with hypophosphatemic rickets/osteomalacia. PLoS One 9: e97830, 2014.

26. Koshida R, Yamaguchi H, Yamasaki K, Tsuchimochi W, Yonekawa $\mathrm{T}$ and Nakazato $\mathrm{M}$ : A novel nonsense mutation in the DMP1 gene in a Japanese family with autosomal recessive hypophosphatemic rickets. J Bone Miner Metab 28: 585-590, 2010.

27. Palmer G, Zhao J, Bonjour J, Hofstetter W and Caverzasio J: In vivo expression of transcripts encoding the Glvr-1 phosphate transporter/retrovirus receptor during bone development. Bone 24: 1-7, 1999.

28. Villa-Bellosta R, Levi M and Sorribas V: Vascular smooth muscle cell calcification and SLC20 inorganic phosphate transporters: Effects of PDGF, TNF-alpha, and Pi. Pflugers Arch 458: 1151-1161, 2009.

29. Brownstein CA, Adler F, Nelson-Williams C, Iijima J, Li P, Imura A, Nabeshima Y, Reyes-Mugica M, Carpenter TO and Lifton RP: A translocation causing increased alpha-klotho level results in hypophosphatemic rickets and hyperparathyroidism. Proc Natl Acad Sci USA 105: 3455-3460, 2008. 